\title{
Neuropilin-1 expression promotes invasiveness of melanoma cells through vascular endothelial growth factor receptor-2- dependent and -independent mechanisms
}

\author{
FEDERICA RUFFINI, STEFANIA D'ATRI and PEDRO M. LACAL \\ Laboratory of Molecular Oncology, Istituto Dermopatico dell'Immacolata-IRCCS, I-00167 Rome, Italy
}

Received November 25, 2012; Accepted January 28, 2013

DOI: $10.3892 /$ ijo.2013.1948

\begin{abstract}
The majority of human melanoma cell lines secretes vascular endothelial growth factor-A (VEGF-A) and expresses its receptors VEGFR-1, VEGFR-2 and neuropilin-1 (NRP-1), a co-receptor for VEGF-A that amplifies the signalling through VEGFR-2. Since it is known that the VEGF-A/VEGFR-2 autocrine loop promotes melanoma cell invasiveness, the aim of the present study was to investigate the involvement of NPR-1 in melanoma progression. Syngeneic human melanoma cell lines expressing either VEGFR-2 or NRP-1, both or none of them, were analyzed for their in vitro ability to migrate, invade the extracellular matrix (ECM) and secrete active metalloproteinase-2 (MMP-2). The results indicate that NRP-1 cooperates with VEGFR-2 in melanoma cell migration induced by VEGF-A. Moreover, NRP-1 expression is sufficient to promote MMP-2 secretion and melanoma cell invasiveness, as demonstrated by the ability of cells expressing solely NRP-1 to spontaneously invade the ECM. This ability is specifically downregulated by anti-NRP-1 antibodies or by interfering with NRP-1 expression using an shRNA construct. Investigation of the signal transduction pathways triggered by NRP-1 in melanoma cells, indicated that NRP-1-dependent promotion of cell invasiveness involves Akt activation through its phosphorylation on T308. Overall, the results demonstrate that NRP-1 is involved in melanoma progression through VEGFR-2-dependent and -independent mechanisms and suggest NRP-1 as a target for the treatment of the metastatic disease.
\end{abstract}

\section{Introduction}

Cutaneous malignant melanoma is the most aggressive form of skin cancer, being endowed with high invasiveness and

Correspondence to: Dr Pedro M. Lacal, Laboratory of Molecular Oncology, Istituto Dermopatico dell'Immacolata-IRCCS, Via Monti di Creta 104, I-00167 Rome, Italy

E-mail: p.lacal@idi.it

Key words: neuropilin-1, VEGFR-2, melanoma, cell invasiveness, metalloproteinase-2 metastatic potential $(1,2)$. Melanoma progression is greatly favoured by angiogenesis and appears to be associated with an increase of vascular endothelial growth factor-A (VEGF-A) expression by the tumour cells (3).

VEGF-A interacts with two high affinity transmembrane tyrosine kinase receptors that are selectively, though not exclusively, expressed by the vascular endothelium (4), namely VEGFR-1 (also known as Flt-1) and VEGFR-2 (or KDR). VEGF-A also binds to neuropilin-1 (NRP-1), a surface polypeptide that, acting as co-receptor, amplifies the signal transmitted by VEGF-A through the VEGFR-2 (5-8).

NRP-1 is a $130-\mathrm{kDa}$ single-spanning transmembrane glycoprotein with a large extracellular domain and a short cytoplasmic tail (44 amino acids), which lacks a defined signalling activity $(5,6)$. It was initially identified as an adhesion molecule in the nervous system. Subsequently, NRP-1 was found to act in neurons as a receptor for class 3 semaphorins (a family of secreted polypeptides with key roles in axonal guidance) and in vascular endothelial cells as a receptor for various members of the VEGF family.

NRP-1 expression has been detected by immunostaining in tumour specimens obtained from patients with prostate, lung, pancreatic or colon carcinoma, but not in the matched normal epithelial tissues. Moreover, NRP-1 is expressed in several other tumours, including melanoma, astrocytoma, malignant glioma and neuroblastoma (reviewed in ref. 5), suggesting a critical role for this receptor in tumour progression (9). Actually, overexpression of NRP-1 has been demonstrated to be positively associated with the aggressiveness and the invasive behaviour of tumour cells $(10,11)$.

The majority of human melanoma cell lines, derived from primary or metastatic lesions, secretes VEGF-A and expresses VEGFR-1, VEGFR-2 and neuropilins (12). Moreover, we previously demonstrated that an autocrine loop sustained by the interaction of VEGF-A with VEGFR-2 promotes melanoma cell ability to migrate and invade the extracellular matrix (ECM) in vitro (13). Therefore, the aim of the present study was, to investigate whether NRP-1 participates as co-receptor in the VEGF-A/VEGFR-2-dependent autocrine loop, contributing to melanoma cell invasiveness. The results indicate that NRP-1 cooperates with VEGFR-2 in promoting melanoma cell migration and ECM invasion. Moreover, NRP-1 expression confers melanoma cells an elevated invasiveness, even in the absence of VEGFR-2 expression. 


\section{Materials and methods}

Reagents. Cell culture media and reagents were purchased from Lonza (Basel, Switzerland); fetal bovine serum (FBS) was from Euroclone (Pero, Italy), heparin, antibiotics and gelatin from Sigma-Aldrich (St. Louis, MO) and fatty acidfree bovine serum albumin (BSA) from Roche (Mannheim, Germany). VEGF-A and polyclonal antibodies used in ELISA (AF-293 and BAF-293), as well as the anti-VEGFR-2 goat polyclonal antibody AF357, were from R\&D Systems (Abingdon, UK). Control rabbit and goat IgGs, were from Sigma-Aldrich. The Akt inhibitor SH-5 [D-3-deoxy-2-O-methyl-myo-inositol 1-[(R)-2-methoxy-3-(octadecyloxy) propyl hydrogen phosphate] was purchased from Enzo Life Sciences (Lausen, Switzerland).

Cell lines and stable transfection of VEGFR-2. The origin and culture of M14 and GR-Mel cell lines have been previously described (12). The isolation and transfection of M14C2 clone with the pcDNA3/VEGFR-2 plasmid (a generous gift of Dr K. Ballmer-Hofer, PSI, Zurich, Switzerland) or with the empty vector (pcDNA3) was performed according to previously described procedures (14). Transfected cells expressing VEGFR-2 were identified by RT-PCR, utilising primers that amplify the fragment of the receptor cDNA sequence comprised between nucleotides 2012 and 2414, as previously described (12).

M14-NV cells were transfected with a pRS plasmid containing a 29-mer shRNA sequence against NRP-1 (TI344368) or with a negative control shRNA pRS plasmid (TR20003), both of them from Origene (Rockville, MD). Transfections were performed as previously described (12) and cell subclones were selected in complete medium supplemented with $2.5 \mu \mathrm{g} /$ $\mathrm{ml}$ puromycin and analyzed for their NRP-1 content by Western blotting.

Real-time quantitative RT-PCR ( $q R T-P C R)$. For each cell line analysed, $1 \mu \mathrm{g}$ of total RNA was reverse-transcribed in 20- $\mu \mathrm{l}$ reaction volume containing Superscript II enzyme (Invitrogen, Paisley, UK) and the following primers: forward, 5'-GTC TAT GCC ATT CCT CCC CC-3'; reverse 5'-GAG ACA GCT TGG CTG GGCT-3'. qRT-PCR was then performed by the duallabelled fluorigenic probe method, using an ABI PRISM 7000 sequence detector (Perkin-Elmer, Groningen, The Netherlands), as previously described (14). Expression levels of VEGFR-2 mRNA were calculated by the relative standard curve method. The level of VEGFR-2 transcript was then normalized to that of 18S RNA and referred to the values of the VEGFR-2-negative M14 cell line, to which the arbitrary value of 1.0 was assigned.

Western blot analysis. NRP-1 expression in the different melanoma cell lines was evaluated in cell extracts by western blot analysis, following a method already described (13). The anti-NRP-1 mouse monoclonal antibody (mAb) A-12 and the anti- $\beta$-tubulin rabbit polyclonal antibody $\mathrm{H}-235$ (used as a loading control) were purchased from Santa Cruz Biotechnology (Santa Cruz, CA) and utilized at the final concentration of $0.2 \mu \mathrm{g} / \mathrm{ml}$.

Evaluation of VEGF-A secretion. Semi-confluent melanoma cell cultures were incubated in $0.1 \%$ BSA/RPMI-1640 medium without FBS for $24 \mathrm{~h}$. Culture supernatants were then collected and concentrated at least ten-fold in Centriplus concentrators (Amicon, Beverly, MA). Cells were detached from the flasks with a solution of $1 \mathrm{mM}$ EDTA in PBS (EDTA/PBS) and the total cell number/culture was recorded. Quantification of the amount of VEGF-A in the concentrated supernatants was performed using Maxisorp Nunc immunoplates (Nunc, Roskilde, Denmark) coated with goat anti-VEGF-A IgGs, as previously described (12).

Migration and invasion assays. In vitro migration assays were performed using Boyden chambers equipped with $8-\mu \mathrm{m}$ pore diameter polycarbonate filters (Nuclepore, Whatman Inc., Clifton, NJ), coated with $5 \mu \mathrm{g} / \mathrm{ml}$ gelatin (13). Briefly, melanoma cells were collected from continuous cultures, washed, suspended in migration medium $(1 \mu \mathrm{g} / \mathrm{ml}$ heparin $/ 0.1 \%$ BSA in RPMI-1640) and loaded ( $2 \times 10^{5}$ cells) into the upper compartment of the Boyden chambers. Migration medium or migration medium containing $20 \mathrm{ng} / \mathrm{ml}$ VEGF-A was added to the lower compartment and the chambers incubated at $37^{\circ} \mathrm{C}$ in a $\mathrm{CO}_{2}$ atmosphere for $18 \mathrm{~h}$ (unless otherwise indicated). The filters were then removed from the chambers, cells fixed in ethanol, stained in crystal violet and those migrated, attached to the lower surface of the filters, counted under the microscope. Twelve high-magnification microscopic fields (magnification $\mathrm{x} 200$ ), randomly selected on triplicate filters, were scored for each experimental condition.

In vitro ECM invasion by melanoma cells was analysed in Boyden chambers as described for the migration assay, but utilising polycarbonate filters coated with $20 \mu \mathrm{g}$ of the commercial basement membrane matrix Matrigel (BD Biosciences, Buccinasco, Italy) instead of gelatin (15) and allowing the cells to invade for $4 \mathrm{~h}$, unless otherwise indicated.

In a set of experiments, migration or invasion assays were performed in the presence of antibodies against NRP-1, VEGFR-2 or matrix metalloproteinase-2 (MMP-2) or the corresponding control immunoglobulins (Igs). Melanoma cells were pre-incubated with the antibody under investigation for $30 \mathrm{~min}$ at room temperature in a rotating wheel. The cells were then loaded in the Boyden chambers without removing the antibody.

Evaluation of MMP-2 secretion. Melanoma cells were seeded into 6 -well plates $\left(2 \times 10^{5}\right.$ cells/well $)$ and allowed to grow near to confluence in complete medium. The cells were then cultured in $2 \mathrm{ml}$ of $0.1 \%$ BSA/RPMI-1640 medium without FBS for $24 \mathrm{~h}$. Afterwards, culture supernatants were collected, centrifuged at $600 \mathrm{x}$ g for $10 \mathrm{~min}$ to remove cells in suspension and frozen at $-20^{\circ} \mathrm{C}$ till use. Cells were detached with EDTA/PBS and counted to determine the total number of cells in the culture. The amount of active MMP- 2 present in the supernatant of the different cell cultures was determined using a human MMP-2 Quantikine immunoassay kit (R\&D Systems).

Phosphorylation profiles of kinases and kinase substrates. A human phospho-kinase array kit (ARY003, from R\&D System) was used to determine in melanoma cells the relative level of phosphorylation of 46 phospho-acceptor sites, identifying a total of 37 polypeptides, comprising kinases and 
kinase substrates. Cell extracts were prepared as indicated by the manufacturer and each part of the array was incubated with an aliquot corresponding to $500 \mu \mathrm{g}$ of protein.

Positive signals were detected on X-ray film by a chemiluminescent reaction, as indicated in the manual of the kit. Relative levels of protein phosphorylation were determined by densitrometric quantification of the spots obtained for each phospho-acceptor site and normalization of the results with respect to the array internal positive controls.

Determination of phospho-Akt (T308) levels. Melanoma cells $\left(2-4 \times 10^{4}\right)$ were cultured in 96 -well plates to near confluence and then fixed in $4 \%$ formaldehyde/PBS for $20 \mathrm{~min}$ at room temperature. Phosphorylation of Akt on T308 was determined using a human phospho-Akt (T308) cell-based ELISA (R\&D Systems). This assay directly quantifies in the cells the amount of Akt phosphorylated on T308 with respect to the total amount of Akt protein.

\section{Results}

Characterization of M14C2 subclones. The human melanoma cell line M14 does not express either VEGFR-2 or VEGFR-1 and displays only low levels of NRP-1 (12). A clone of this cell line (i.e., M14C2) was, therefore, utilized to investigate whether NRP-1 expression participates in the VEGF-A/VEGFR-2 autocrine loop that promotes melanoma cell invasiveness (13). M14C2 cells were transfected with a plasmid containing the cDNA encoding VEGFR-2 or with the empty vector and subclones expressing this receptor and/ or NRP-1, or negative for both polypeptides, were identified. Based on the expression levels of NRP1 and VEGFR-2, four subclones were then selected: one subclone obtained from M14C2 cells transfected with the empty vector, namely M14-C, which does not express either VEGFR-2 or NRP-1; three subclones obtained from M14C2 cells transfected with the VEGFR-2 containing plasmid, namely M14-NV, which expresses both receptors, M14-N, which expresses only NRP-1 at levels comparable to those displayed by the M14-NV subclone and M14-V, which expresses only VEGFR-2 at levels comparable to those displayed by the M14-NV subclone (Fig. 1A and B).

Melanoma cells expressing either NRP-1 alone or VEGFR-2 alone displayed a 2-fold increase in the levels of VEGF-A secretion as compared with the cells negative for both receptors (Fig. 1C). Moreover, the simultaneous expression of VEGFR-2 and NRP-1 was accompanied by a further enhancement of VEGF-A secretion by melanoma cells (Fig. 1C).

The analysis of the ability of the four transfected subclones to spontaneously invade the ECM revealed that the cells expressing only NRP-1 were endowed with higher invasiveness as compared to the cells devoid of both NRP-1 and VEGFR-2 or expressing only the latter receptor (Fig. 1D). Moreover, the cells co-expressing VEGFR-2 and NRP-1 showed an invasive capacity significantly higher than that displayed by the cells expressing only NRP-1 (Fig. 1D).

The transfected subclones were also analyzed for the ability to migrate under basal conditions or in response to VEGF-A. Higher basal levels of migration were observed in NRP-1 expressing cells with respect to the cells negative
A
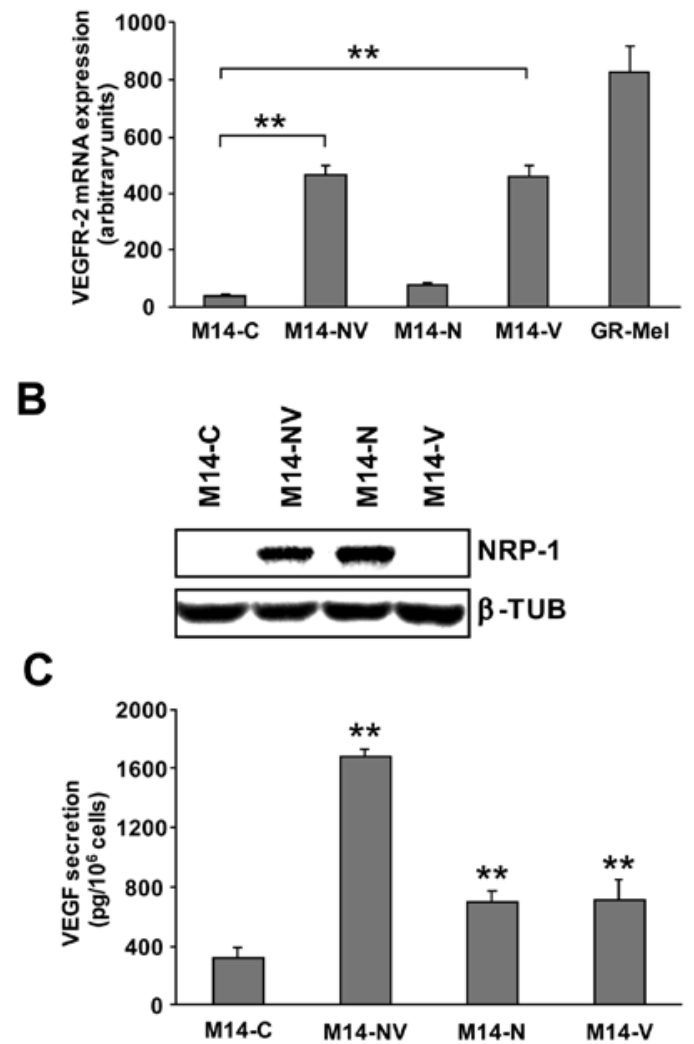

D

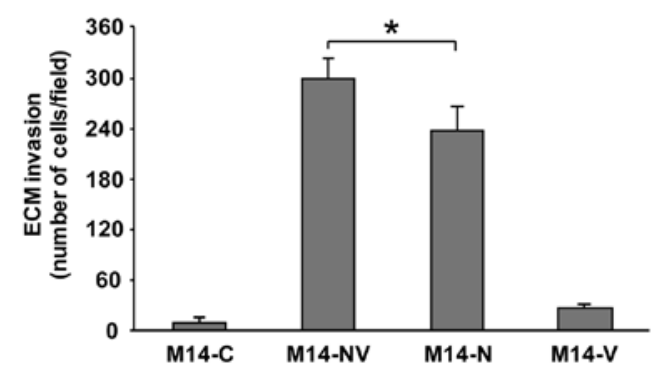

Figure 1. Characterization of transfected M14C2 subclones. (A) VEGFR-2 expression in the selected subclones was determined by qRT-PCR, as described in Materials and methods. Each histogram represents the mean value of at least three independent determinations, with bars indicating SD of the mean. RNA from the melanoma cell line GR-Mel was used as a positive control in the same assay. ${ }^{* *} \mathrm{P}<0.01$, according to Student's t-test analysis. (B) The expression of NRP-1 in the selected subclones was evaluated by western blot analysis as described in Materials and methods, loading $80 \mu \mathrm{g}$ of proteins per sample on a $7 \%$ SDS-polyacrylamide gel and utilising antibodies against NRP-1 or against $\beta$-tubulin as a loading control. The results are representative of two independent experiments. (C) VEGF-A secretion by the different subclones was quantified by ELISA as indicated in Materials and methods. Each value represents the mean \pm SD of at least three independent determinations. ${ }^{* *} \mathrm{P}<0.01$, according to Student's t-test analysis comparing the amount of VEGF-A secreted by M14-NV, M14-N or M14-V cells with that secreted by M14-C. (D) Spontaneous invasion of ECM by the different subclones was evaluated as described in Materials and methods, using Boyden chambers equipped with Matrigel-coated filters. Data are expressed in terms of number of invading cells per microscopic field. Each value represents the mean \pm SD of three independent experiments, in which 12 microscopic fields on triplicate filters were scored for each experimental condition. ${ }^{*} \mathrm{P}<0.05$, according to Student's t-test analysis.

for both NRP-1 and VEGFR-2 or expressing only the latter receptor (Table I). As expected, only the subclones expressing VEGFR-2 displayed a chemotactic response to VEGF-A. 
A

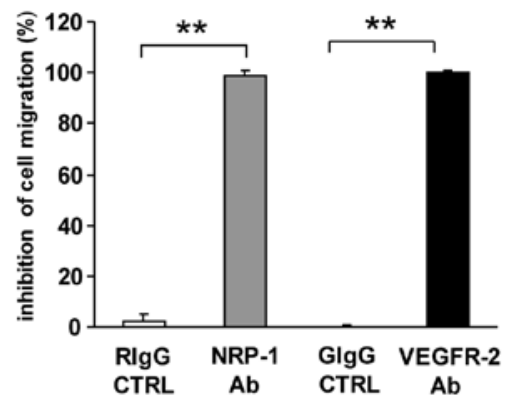

B

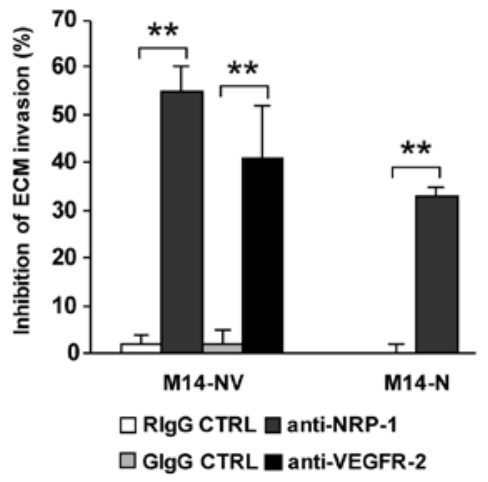

C

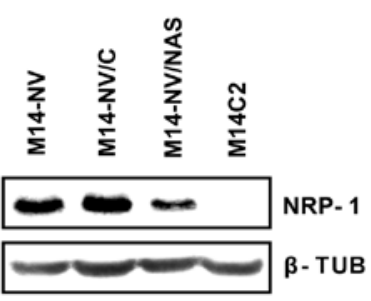

D

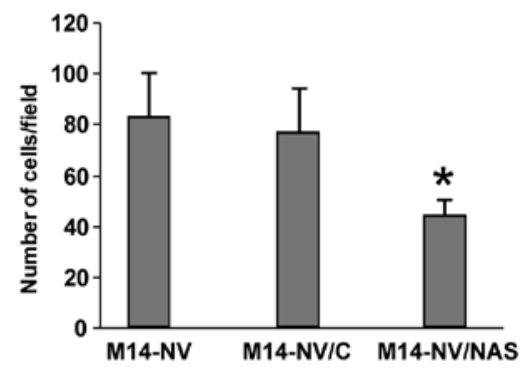

$\mathbf{E}$

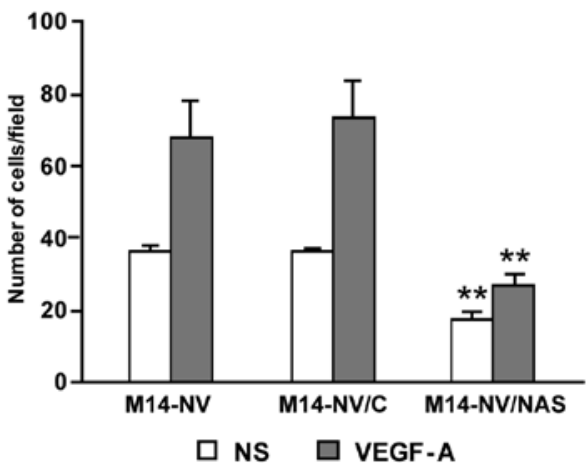

Figure 2. NRP-1 and VEGFR-2 cooperate in the promotion of melanoma cell migration and ECM invasion. (A) Migration of M14-NV cells in response to VEGF-A $(20 \mathrm{ng} / \mathrm{ml})$ was tested in the presence of $5 \mu \mathrm{g} / \mathrm{ml}$ of rabbit polyclonal anti-NRP-1 (H-286, from Santa Cruz Biotechnology) or goat polyclonal anti-VEGFR-2 antibodies (AF357, from R\&D Systems) or their corresponding control IgG [i.e., rabbit IgG (RIgG CTRL) and goat IgG (GIgG CTRL)], or in the absence of antibodies. Data are expressed in terms of percentage of migration inhibition of antibody-treated cells with respect to untreated cells. Each value represents the mean $\pm \mathrm{SD}$ of at least three independent experiments. ${ }^{* *} \mathrm{P}<0.01$, according to Student's $\mathrm{t}$-test analysis. (B) The ability of M14-NV cells to spontaneously invade the ECM was tested in the absence or in the presence of the antibodies indicated in (A) and as described in Materials and methods. The effects of anti-NRP-1 antibody and the corresponding control IgG on M14-N cell invasiveness were also evaluated. Data are expressed in terms of percentage of ECM invasion inhibition of antibody-treated cells with respect to untreated cells. Each value represents the mean \pm SD of at least three independent experiments. ${ }^{* *} \mathrm{P}<0.01$, according to Student's t-test analysis. (C) The expression of NRP-1 in the selected subclones was evaluated by western blot analysis as described in Materials and methods, loading $80 \mu \mathrm{g}$ of proteins per sample on a 7\% SDS-polyacrylamide gel and utilising antibodies against NRP-1 or against $\beta$-tubulin as a loading control. Extracts from M14C2 parental cells were used as negative control. The results are representative of two independent experiments. (D) Spontaneous invasion of ECM by the different subclones was evaluated as described in Materials and methods, using Boyden chambers equipped with Matrigel-coated filters and incubation time of $2 \mathrm{~h}$. Data are expressed in terms of number of invading cells per microscopic field. Each value represents the mean \pm SD of three independent experiments, in which 12 microscopic fields on triplicate filters were scored for each experimental condition. ${ }^{*} \mathrm{P}<0.05$, according to Student's t-test analysis comparing the invasive ability of M14-NV/NAS cells with that of M14-NV cells. (E) Migration of the different subclones in response to VEGF-A (20 ng/ml) or in non-stimulating conditions (NS) was evaluated as described in Materials and methods, using Boyden chambers equipped with gelatin-coated filters and incubation time of $5 \mathrm{~h}$. Data are expressed in terms of number of migrated cells. Each value represents the mean $\pm \mathrm{SD}$ of at least three independent experiments. ${ }^{* *} \mathrm{P}<0.01$, according to Student's t-test analysis comparing migration of M14-NV/NAS cells with that of M14-NV cells.

Involvement of NRP-1 in melanoma cell migration and ECM invasion. The involvement of NRP-1 expression in melanoma cell ability to migrate and invade the ECM was further investigated using an anti-NRP-1 antibody against the MAM (meprin, A5, $\mu$-phosphatase) domain of this polypeptide, reported to be involved in the receptor oligomerization $(16,17)$. Treatment with this antibody drastically reduced the migratory response of M14-NV cells to VEGF-A (Fig. 2A). Comparable results were obtained using an anti-VEGFR-2 antibody that blocks VEGF-A binding to this receptor (Fig. 2A). The anti-NRP-1 antibody also inhibited M14-NV and M14-N cell ability to spontaneously invade the ECM (a 55 and 33\% inhibition, respectively) (Fig. 2B). As expected, the anti-VEGFR-2 blocking antibody downmodulated M14-NV cell invasiveness ( $41 \%$ inhibition) (Fig. 2B).

Downmodulation of NRP-1 expression in M14-NV cells was achieved by transfection of these cells with a pRS plasmid containing a specific 29-mer shRNA sequence (M14-NV/ 
Table I. Migratory capability of the different M14C2 subclones in response to VEGF-A.

\begin{tabular}{lccc}
\hline $\begin{array}{l}\text { Cell } \\
\text { subclone }\end{array}$ & $\begin{array}{c}\text { Non- } \\
\text { stimulated }^{\mathrm{a}}\end{array}$ & $\begin{array}{c}\text { VEGF-A } \\
\text { stimulation }^{\mathrm{a}}\end{array}$ & \begin{tabular}{c} 
Migration $_{\text {index }^{\mathrm{b}}}$ \\
\hline M14-C
\end{tabular} \\
$3 \pm 1$ & $3 \pm 1$ & 1.00 \\
M14-NV & $165 \pm 29$ & $251 \pm 18$ & 1.52 \\
M14-N & $114 \pm 28$ & $117 \pm 32$ & 1.02 \\
M14-V & $10 \pm 3$ & $34 \pm 3$ & 3.40 \\
\hline
\end{tabular}

${ }^{a}$ Migration assays were performed in Boyden chambers equipped with gelatin coated polycarbonate filters, in the absence or in the presence of VEGF-A (20 ng/ml in migration medium) as stimulus. Values represent the number of migrated cells per microscopic field (magnification $\mathrm{x} 200$ ) and are the mean of at least three independent determinations $\pm \mathrm{SD} .{ }^{\mathrm{b}}$ The migration index was calculated as the ratio between the number of cells/microscopic field in the experimental condition analysed and the number of cells/microscopic field in the basal condition (i.e., in the absence of any stimulus). The migration index in the basal condition corresponds to 1 .

NAS subclone). To generate a suitable negative control cell line, M14-NV cells were also transfected with an empty pRS plasmid (M14-NV/C subclone). Downmodulation of NRP-1 expression in M14-NV/NAS cells resulted in 49\% reduction of NRP-1 protein levels with respect to the control cells (Fig. 2C). M14-NV/NAS cells showed a $46 \%$ decrease in the ability to invade the ECM (Fig. 2D). Moreover, their ability to migrate either in response to VEGF-A or in non-stimulating conditions was abrogated or halved, respectively (Fig. 2E).

MMP-2 secretion by M14C2 subclones. In an attempt to shed light on the mechanism by which NRP-1 promotes melanoma cell invasiveness, secretion of the metalloproteinase MMP-2 by the different transfected subclones was analyzed. M14-NV and M14-N cells showed levels of MMP-2 secretion 6-fold higher than those displayed by M14-V or M14-C cells (Fig. 3A), strongly suggesting that NRP-1 expression up-modulates the secretion of this metalloproteinase by human melanoma cells. Moreover, in vitro ECM invasion by M14-NV and M14-N cells was inhibited in the presence of an anti-MMP-2 antibody, confirming the involvement of this metalloproteinase in the elevated invasiveness of NRP-1 expressing cells (Fig. 3B).

Involvement of Akt signalling pathway in the invasiveness of NRP-1 expressing melanoma cells. The results obtained in the functional assays described above, suggested that NRP-1 might activate in melanoma cells signal transduction pathways independently of its interaction with VEGFR-2. With the intent to identify any of these signal transduction pathways, the constitutive phosphorylation status of a set of kinases and kinase substrates was determined in NRP-1 expressing cells with respect to NRP-1 negative cells, using a human phosphokinase array kit.

The results indicated that the phosphorylation status of several proteins was different between NRP-1-positive and NRP-1-negative cells (Table II). The phospho-acceptor sites
A
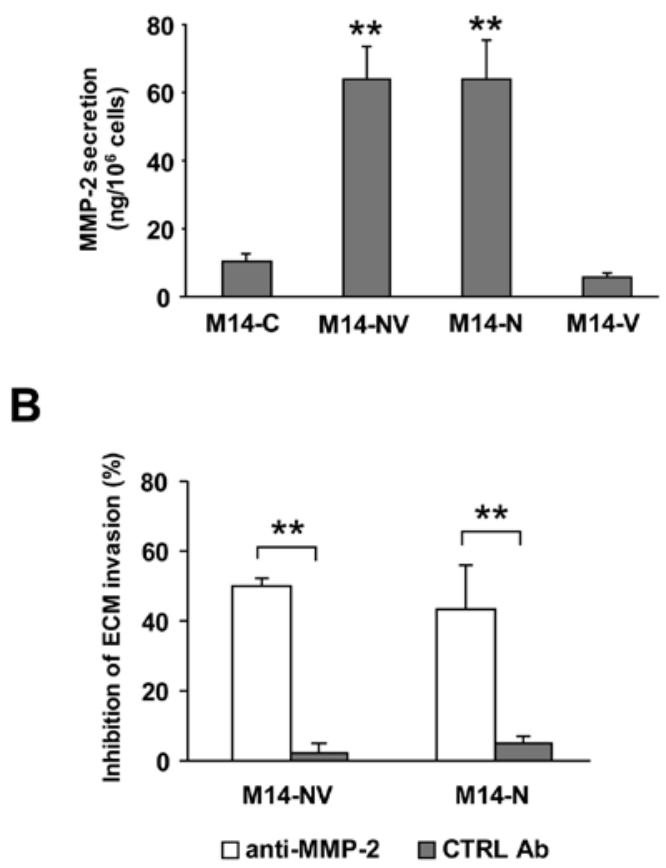

Figure 3. Increased secretion of MMP-2 is involved in NRP-1-induced ECM invasion by melanoma cells. (A) MMP-2 secretion by the transfected subclones was quantified in culture cell supernatants by ELISA as described in Materials and methods. Each value represents the mean \pm SD of at least three independent determinations. ${ }^{* *} \mathrm{P}<0.01$, according to Student's t-test analysis comparing the amount of MMP-2 secreted by M14-NV or by M14-N cells with that secreted by M14-C cells. (B) The ability of M14-NV and M14-N cells to spontaneously invade the ECM was tested in the presence of $3 \mu \mathrm{g} / \mathrm{ml}$ of an anti-MMP-2 antibody or a control antibody (goat IgG, CTRL Ab), or in the absence of antibodies, as described in Fig. 2. Data are expressed in terms of percentage of inhibition of ECM invasion by antibody-treated cells with respect to untreated cells. Each value represents the mean \pm SD of at least three independent experiments. ${ }^{* *} \mathrm{P}<0.01$, according to Student's t-test analysis.

that showed an increased phosphorylation level of $\geq 2$-fold in M14-NV and M14-N cells with respect to M14-C and M14-V cells, are shown in Fig. 4A. The highest increase in the level of phosphorylation was observed on T308 of Akt [phosphoAkt (T308)] and S63 of c-Jun. A remarkable increase in the level of phosphorylation was also detected on S15 of p53, T421/S426 and T229 of the ribosomal S6 kinases p70S6K, S380 and S221 of the ribosomal S6 kinases RSK and Y693 of STAT4 (Fig. 4A).

On the other hand, only four kinases displayed a significant reduction of phosphorylation levels in NRP-1 expressing cells, namely the mammalian target of rapamycin (mTOR) and three members of the Scr family of non-receptor tyrosine kinases (Src, Fyn and Hck) (Fig. 4B).

Aberrant activation of the PI3K/Akt signalling pathway has been implicated in melanoma onset, progression and chemoresistance $(18,19)$. Thus, studies were performed to investigate the possible role of increased Akt phosphorylation in M14-NV and M14-N cell invasiveness. To this end, the levels of phospho-Akt (T308) in the four transfected subclones were determined by a cell-based ELISA method, which confirmed the results obtained with the phosphokinase 
Table II. Phosphorylation levels of a set of kinases and kinase substrates in the different transfected M14C2 subclones.

\begin{tabular}{|c|c|c|c|c|}
\hline \multirow[b]{2}{*}{ Protein (phosphorylation site) } & \multicolumn{4}{|c|}{ Relative level of phosphorylation ${ }^{a}$} \\
\hline & M14-C & M14-NV & M14-N & M14-V \\
\hline $\mathrm{p} 38 \alpha(\mathrm{T} 180 / \mathrm{Y} 182)$ & 70 & 97 & 91 & 32 \\
\hline ERK1/2 (T202/Y204, T185/Y187) & 80 & 81 & 100 & 13 \\
\hline JNK pan (T183/Y185, T221/Y223) & 10 & 96 & 140 & 71 \\
\hline GSK-3 $\alpha / \beta(\mathrm{S} 21 / \mathrm{S} 9)$ & 9 & 130 & 130 & 130 \\
\hline MEK1/2 (S218/S222, S222/S226) & 140 & 90 & 110 & 71 \\
\hline MSK1/2 (S376/S360) & 140 & 83 & 170 & 38 \\
\hline AMPK $\alpha 1$ (T174) & 40 & 140 & 250 & 93 \\
\hline Akt (S473) & 0 & 73 & 100 & 110 \\
\hline TOR (S2448) & 170 & 100 & 99 & 180 \\
\hline CREB (S2448) & 460 & 310 & 450 & 480 \\
\hline HSP27 (S78/S82) & 84 & 54 & 140 & 31 \\
\hline AMPK $\alpha 2$ (T172) & 60 & 110 & 150 & 73 \\
\hline$\beta$-catenin & 23 & 130 & 170 & 91 \\
\hline Src (Y419) & 300 & 220 & 210 & 320 \\
\hline Lyn (Y397) & 210 & 110 & 110 & 130 \\
\hline Lck (Y394) & 53 & 28 & 67 & 16 \\
\hline STAT2 (Y689) & 96 & 120 & 180 & 100 \\
\hline STAT5a (Y699) & 260 & 240 & 330 & 340 \\
\hline Fyn (Y420) & 200 & 140 & 70 & 220 \\
\hline Yes (Y426) & 300 & 250 & 230 & 270 \\
\hline Fgr (Y412) & 90 & 81 & 110 & 41 \\
\hline STAT3 (Y705) & 11 & 54 & 90 & 6 \\
\hline STAT5b (Y699) & 330 & 290 & 330 & 430 \\
\hline Hck (Y411) & 160 & 130 & 38 & 180 \\
\hline Chk-2 (T68) & 240 & 210 & 140 & 330 \\
\hline FAK (Y397) & 23 & 38 & 60 & 13 \\
\hline STAT6 (Y641) & 73 & 120 & 150 & 60 \\
\hline STAT5a/b (Y699) & 2 & 100 & 83 & 78 \\
\hline p53 (S392) & 1,230 & 1,420 & 1,290 & 1,170 \\
\hline Akt (T308) & 14 & 380 & 420 & 65 \\
\hline p53 (S46) & 1,060 & 1,160 & 1,190 & 1,000 \\
\hline P70 S6 kinase (T389) & 0 & 46 & 97 & 0 \\
\hline p53 (S15) & 80 & 230 & 260 & 62 \\
\hline p27 (T198) & 4 & 27 & 36 & 0 \\
\hline Paxillin (Y118) & 110 & 190 & 220 & 83 \\
\hline P70 S6 kinase (T421/S424) & 71 & 220 & 310 & 31 \\
\hline RSK 1/2/3 (S380) & 80 & 230 & 300 & 43 \\
\hline p27 (T157) & 17 & 66 & 64 & 0 \\
\hline PLC $\gamma-1(Y 783)$ & 90 & 160 & 140 & 51 \\
\hline P70 S6 kinase (T229) & 90 & 160 & 290 & 17 \\
\hline RSK 1/2 (S221) & 45 & 130 & 230 & 8 \\
\hline c-Jun (S63) & 54 & 370 & 550 & 22 \\
\hline Pyk2 (Y402) & 22 & 70 & 60 & 9 \\
\hline STAT1 (Y701) & 68 & 110 & 250 & 34 \\
\hline STAT4 (Y693) & 20 & 60 & 170 & 0 \\
\hline eNOS (S1177) & 120 & 200 & 260 & 54 \\
\hline
\end{tabular}

${ }^{a}$ The phosphorylation level of the indicated proteins was simultaneously analyzed in cell extracts from the different transfected subclones using a human phospho-kinase array kit, as described in Materials and methods. Relative levels of protein phosphorylation were determined by densitometric quantification of the spots obtained and normalization of the results with respect to the internal positive controls. Data refer to a representative experiment performed with duplicate samples. 
A

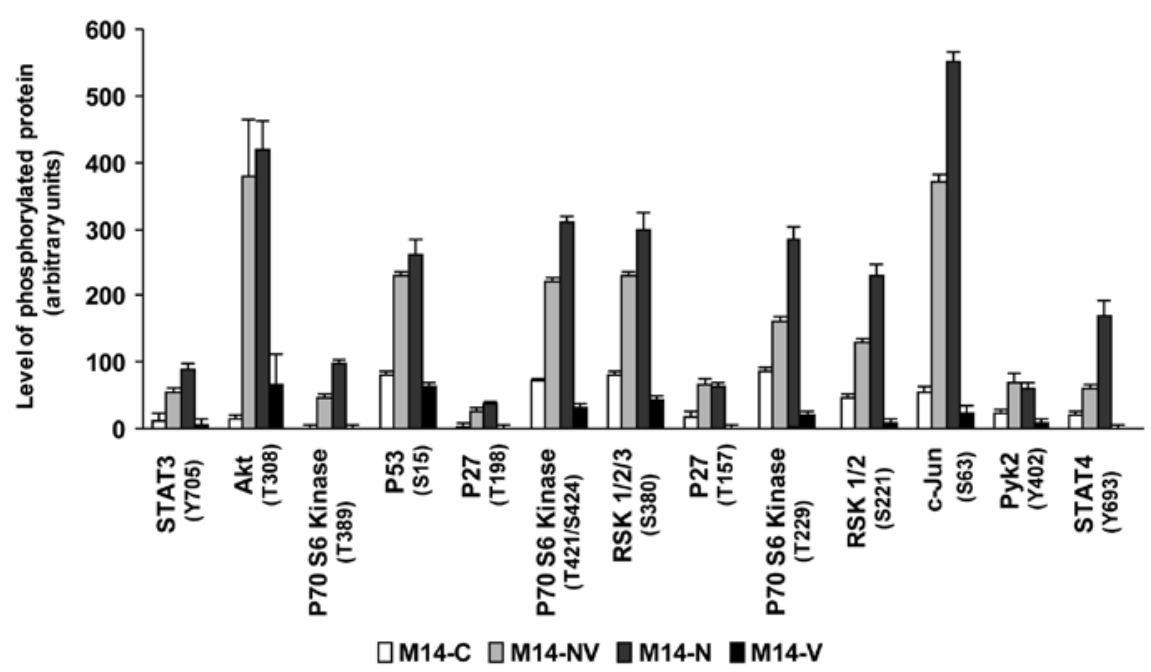

B

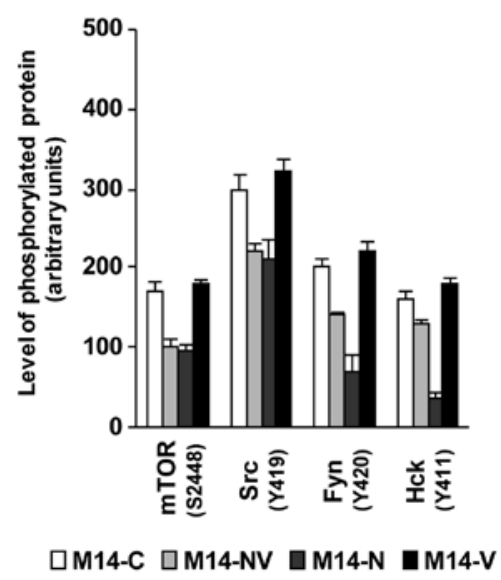

Figure 4. Identification of signal transduction pathways modulated by NRP-1 expression in melanoma cells. The level of phosphorylation of 37 proteins represented by kinases and kinase substrate, was determined in cell extracts of the different transfected subclones using a phospho-kinase array kit, as described in Materials and methods. Data refer to a representative experiment performed with duplicate samples and each value corresponds to the mean \pm SD. (A) Protein sites for which phosphorylation was at least 2-fold higher in NRP-1 expressing subclones (M14-NV and M14-N) than in NRP-1 negative subclones (M14-C and M14-V). (B) Sites for which phosphorylation was lower in NRP-1 expressing subclones than in NRP-1 negative subclones.

array (Fig. 5A). Actually, untreated M14-NV and M14-N cells expressed higher levels of phospho-Akt (T308) as compared with the NRP-1-negative cells. Using the same immunoassay, the effects of a specific Akt inhibitor (SH-5), which prevents phosphatidylinositol-trisphosphate binding to this kinase (20), were also investigated in M14-NV and M14-N cells. As expected, melanoma cell exposure to $\mathrm{SH}-5$ caused a significant reduction in the levels of phosphorylated Akt (Fig. 5A). Moreover, pre-treatment of M14-NV and M14-N cells with SH-5 also resulted in a marked downmodulation of cell invasive capacity (Fig. 5B), strongly suggesting that the activation of Akt contributes to the higher invasiveness of melanoma cells expressing NRP-1.

\section{Discussion}

NRP-1, when co-expressed with VEGFR-2, is considered a VEGF-A binding co-receptor, amplifying not only endothelial cell but also tumour cell response to VEGF-A. The results of the present study demonstrate that NRP-1 plays an important role in the regulation of melanoma cell ability to migrate and invade the ECM when expressed in cells also positive for VEGFR-2. Indeed, spontaneous in vitro ECM invasion by M14-NV cells expressing both NRP-1 and VEGFR-2, was significantly higher than that of M14-V cells expressing solely VEGFR-2 and was downmodulated not only by antibodies specific for VEGFR-2 but also by anti-NRP-1 antibodies and NRP-1 knockdown. Non-stimulated and VEGF-A-stimulated M14-NV cells also showed a higher level of in vitro migration as compared with M14-V cells, either non-stimulated or exposed to VEGF-A. Notably, the chemotactic response to VEGF-A of M14-NV cells was almost abrogated not only by antibodies specific for VEGFR-2 but also by anti-NRP-1 antibodies and NRP-1 knockdown. This finding demonstrates that NRP-1, acting as a co-receptor for VEGF-A, co-operates with VEGFR-2 to promote melanoma cell migration in response to 
A

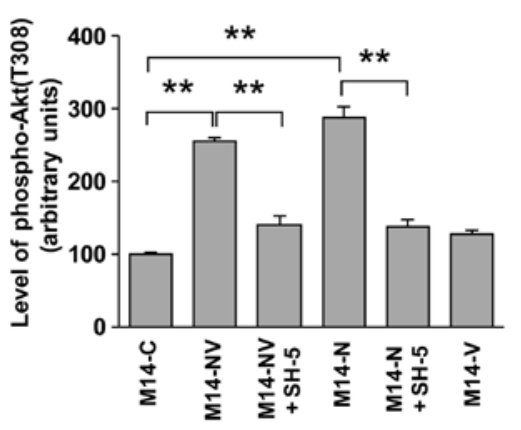

B

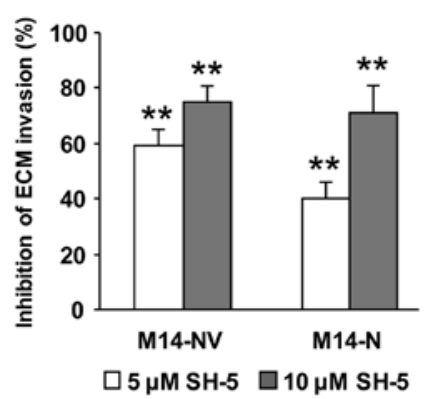

Figure 5. The Akt inhibitor SH-5 impairs Akt phosphorylation on T308 and ECM invasion by M14-NV and M14-N cells. (A) The level of Akt phosphorylated on T308, normalized to total Akt, was determined in the different transfected subclones by a cell-based ELISA, as indicated in Materials and methods. Where indicated, cell cultures were pre-treated with the Akt inhibitor SH-5 $(10 \mu \mathrm{M})$ for $2 \mathrm{~h}$ before determination of Akt phosphorylation. Data refer to a representative experiment performed with quadruplicate samples. Each value represents the mean $\pm \mathrm{SD} .{ }^{* *} \mathrm{P}<0.01$, according to Student's t-tes analysis. (B) M14-NV and M14-N cells were incubated with 5 or $10 \mu \mathrm{M} \mathrm{SH}-5$ for $2 \mathrm{~h}$ and then loaded, without removing the inhibitor, in the upper compartments of Boyden chambers equipped with Matrigel-coated filters. ECM invasion was then assayed as indicated in Materials and methods, for $2 \mathrm{~h}$ Data are expressed in terms of percentage of inhibition of ECM invasion by SH-5-treated cells with respect to untreated cells. Each value represents the mean $\pm \mathrm{SD}$ of at least three independent experiments. ${ }^{* *} \mathrm{P}<0.01$, according to Student's t-test analysis comparing ECM invasion by SH5-treated M14-NV or M14-N cells with that of untreated cells.

this cytokine, as previously described in other tumour types $(5,7)$. Moreover, melanoma cells expressing both VEGFR-2 and NRP-1 produce VEGF-A amounts significantly higher than those secreted by the cells expressing only VEGFR-2. This feature might enhance tumour angiogenesis and sustain the VEGF-A/VEGFR-2 autocrine loop in vivo, leading to increased tumour growth and metastases.

Considerable experimental evidence has suggested that the intracellular domain of NRP-1 possesses the ability to activate signal transduction pathways also independently of VEGFR-2 tyrosine kinase activity $(21,22)$. Actually, the three $\mathrm{C}$-terminal residues of NRP-1 allow the interaction of this polypeptide with protein adapters that contain the PDZ (PSD-95/Dlg/ ZO-1) domain that could connect NRP-1 with intracellular signalling molecules (23). An example of such kind of proteins is synectin, an important modulator of arterial development and branching through its involvement in the regulation of cell polarization and migration (24).

Consistent with the ability of NRP-1 to activate signal transduction pathways independent of VEGFR-2, the results of our study show that the expression of NRP-1 is also able to promote cell migration and ECM invasion in the absence of VEGFR-2 co-expression. Indeed, M14-N cells displayed elevated levels of spontaneous in vitro migration and ECM invasion as compared not only with M14-C cells, negative for both NRP-1 and VEGFR-2, but also with M14-V cells. Both biological processes are activated during tumour metastatic spreading and depend on the degradation and remodelling of the ECM and basement membranes by proteolytic enzymes. In particular, MMP-2 expression has been correlated with melanoma progression and it represents an unfavourable prognostic factor for this type of cancer $(25,26)$. Our results demonstrate that the NRP-1 expression is by itself sufficient to upregulate active MMP-2 secretion and that the activity of this enzyme is essential for ECM invasion by melanoma cells, as described for other tumour types (27).

In an attempt to identify signal transduction pathways activated by NRP-1 which might promote melanoma cell invasiveness, the phosphorylation status of a set of 37 different polypeptides (comprising kinases and kinase substrates) was comparatively analyzed in NRP-1-positive and NPR-1-negative cells. A marked increase in the phosphorylation of the transcription factor c-Jun at the $\mathrm{S} 63$ site and of the serine/threonine kinase Akt, at the T308 site was observed in NRP-1 expressing cells. c-Jun is known to be implicated in the activation of MMP expression (28). On the other hand, Akt activation has been shown to induce ECM invasion and MMP-2 secretion in epithelial cells (29) and phospho-Akt expression in melanoma specimens has been found to increase with disease progression and to inversely correlate with patient survival (18). Thus, simultaneous activation of Akt and c-Jun in melanoma cells expressing NRP-1 might co-operate to induce MMP-2 secretion and promote ECM invasion. This hypothesis is supported by the observation that inhibition of Akt activity resulted in a remarkable decrease of ECM invasion by melanoma cells expressing solely the NRP-1.

The increased levels of phospho-Akt (T308) in NRP-1 expressing cells might be the result of the reduced phosphorylation of mTOR observed in these cells. The mTOR protein kinase exists in two complexes, mTORC1 and mTORC2, depending on the proteins to which it associates. mTORC1, which is activated downstream of Akt in the PI3K/Akt pathway, phosphorylates two major substrates, namely p70S6K and 4E-BP1. Phosphorylation of p70S6K on T389 causes a feedback inhibition of the receptor tyrosine kinases responsible for Akt phosphorylation (30). Moreover, mTORC1 phosphorylates and activates also the protein phosphatase 2 (PP2A), which dephosphorylates p53 at Ser15 (31) and Akt at T308 (32). mTORC2 complex has been shown to directly phosphorylate Akt and SGK1. In particular, phosphorylation of Akt on S473 by mTORC2 enhances the catalytic activity of Akt already phosphorylated on T308 by the serine/threonine kinase PDK1 (phosphoinositide-dependent kinase 1). In this context, mTOR kinase activity downmodulation and low levels of p70S6K phosphorylation on T389 result in the relieve of receptor tyrosine kinases feedback inhibition by mTORC1 complex, leading to an increase of Akt phosphorylation even if mTORC2 activity is reduced $(33,34)$. Interestingly, it has been demonstrated that mTORC1 activity down-modulation results in Akt phosphorylation on T308, but not on S473 and 
that phospho-Akt (T308) is active and able to phosphorylate key substrates of the PI3K/Akt signal transduction pathway (35). These data might explain why upregulation of phosphoAkt (T308) in melanoma cells expressing NRP-1 results in the promotion of cell invasiveness in the absence of a parallel upregulation of Akt phosphorylation on S473.

Our study demonstrates that in melanoma cells expressing NRP-1 mTOR phosphorylation is reduced with respect to NRP-1 negative cells and that phosphorylation of p70S6K on T389 in these cells is also low, as compared to p70S6K phosphorylation on T421/S424 or T229. Moreover, consistent with the literature findings, NRP-1 expressing cells display increased levels not only of phospho-Akt (T308), but also of phospho-p53 (S15). Therefore, our results suggest that the expression of NRP-1 in melanoma cells might favour the up-modulation of a signal transduction pathway that involves phospho-Akt (T308) and might result in the promotion of migratory and invasive capabilities in these cells.

In addition, phosphorylation of two ribosomal S6 kinases was found to be down-modulated in NRP-1 expressing cells, namely p70S6K and RSK. p70S6K, besides having the abovementioned role in the negative feedback induced by mTOR, when phosphorylated by different kinases on other sites, such as the T229, might act as a positive regulator of melanoma cell invasiveness (36). RSK is activated through the ERK pathway (37) and has been shown to be an important common effector for multiple migratory stimuli (38-40). Both RSK and p70S6K contain PDZ regions $(36,37)$ which might interact with the carboxi-terminus end of NRP-1, in a way similar to that already described for synectin (24). NRP-1 might therefore recruit $\mathrm{S} 6$ kinases to the membrane and favour their activation by PDK1 that, besides phosphorylating Akt, is also able to activate p70S6K and RSK (41). NRP-1 might, in this way, positively modulate both Akt and ERK signalling pathways.

Cutaneous malignant melanoma is endowed with high invasiveness and metastatic potential as well as with elevated chemo- and radioresistance. During melanoma progression, the transition from the radial growth phase (RGP) to the vertical growth phase (VGP) is a key event, given that RGP melanomas are mostly curable by surgical resection, whereas VGP melanomas have the ability to metastasize $(2,5)$. This transition seems to be associated with an increase of VEGF-A expression by the tumour cells (3). In this context, the results herein presented demonstrate that NRP-1 plays a critical role in melanoma cell migration sustained by the VEGF-A/ VEGFR-2 autocrine loop. Moreover, they provide for the first time evidence that NRP-1 expression strongly promotes melanoma cell invasiveness also in the absence of VEGFR-2 and that this effect depends, at least in part, on Akt activation and on enhanced secretion of MMP-2. A better understanding of the mechanisms by which NRP-1 regulates melanoma invasive capacity might help to design novel and more effective strategies, including the development of NRP-1 antagonists, for the treatment of the metastatic disease, that presently has few therapeutic options.

\section{Acknowledgements}

We acknowledge Professor Grazia Graziani (Pharmacology and Medical Oncology Section, Department of System
Medicine, University of Rome 'Tor Vergata', Rome, Italy) for critical reading of the manuscript and Daniele Bartoloni for the artwork. This study was supported by the Italian Ministry of Health.

\section{References}

1. Danson S and Lorigan P: Improving outcomes in advanced malignant melanoma: update on systemic therapy. Drugs 65: 733-743, 2005

2. Miller AJ and Mihm MC Jr: Melanoma. N Engl J Med 355: 51-65, 2006.

3. Marcoval J, Moreno A, Graells J, Vidal A, Escriba JM, GarciaRamirez M and Fabra A: Angiogenesis and malignant melanoma. Angiogenesis is related to the development of vertical (tumorigenic) growth phase. J Cutan Pathol 24: 212-218, 1997.

4. Herold-Mende C, Steiner H H andl T, et al: Expression and functional significance of vascular endothelial growth factor receptors in human tumor cells. Lab Invest 79: 1573-1582, 1999.

5. Pellet-Many C, Frankel P, Jia H and Zachary I: Neuropilins: structure, function and role in disease. Biochem J 411: 211-226, 2008.

6. Geretti E, Shimizu A and Klagsbrun M: Neuropilin structure governs VEGF and semaphorin binding and regulates angiogenesis. Angiogenesis 11: 31-39, 2008.

7. Grünewald FS, Prota AE, Giese A and Ballmer-Hofer K: Structure-function analysis of VEGF receptor activation and the role of coreceptors in angiogenic signaling. Biochim Biophys Acta 1804: 567-580, 2010.

8. Bagri A, Tessier-Lavigne M and Watts RJ: Neuropilins in tumor biology. Clin Cancer Res 15: 1860-1864, 2009.

9. Ellis LM: The role of neuropilins in cancer. Mol Cancer Ther 5: 1099-1107, 2006

10. Latil A, Bieche I, Pesche S, Valeri A, Fournier G, Cussenot O and Lidereau R: VEGF overexpression in clinically localized prostate tumors and neuropilin-1 overexpression in metastatic forms. Int J Cancer 89: 167-171, 2000.

11. Hansel DE, Wilentz RE, Yeo CJ, Schulick RD, Montgomery E and Maitra A: Expression of neuropilin-1 in high-grade dysplasia, invasive cancer and metastases of the human gastrointestinal tract. Am J Surg Pathol 28: 347-356, 2004.

12. Lacal PM, Failla CM, Pagani E, et al: Human melanoma cells secrete and respond to placenta growth factor and vascular endothelial growth factor. J Invest Dermatol 115: 1000-1007, 2000.

13. Lacal PM, Ruffini F, Pagani E and D'Atri S: An autocrine loop directed by the vascular endothelial growth factor promotes invasiveness of human melanoma cells. Int J Oncol 27: 1625-1632, 2005.

14. Ruffini F, Failla CM, Orecchia A, et al: Expression of the soluble vascular endothelial growth factor receptor-1 in cutaneous melanoma: role in tumour progression. Br J Dermatol 164: 1061-1070, 2011.

15. Lacal PM, Morea V, Ruffini F, et al: Inhibition of endothelial cell migration and angiogenesis by a vascular endothelial growth factor receptor-1 derived peptide. Eur J Cancer 44: 1914-1921, 2008.

16. Chen H, He Z, Bagri A and Tessier-Lavigne M: Semaphorinneuropilin interactions underlying sympathetic axon responses to class III semaphorins. Neuron 21: 1283-1290, 1998.

17. Nakamura F, Tanaka M, Takahashi T, Kalb RG and Strittmatter SM: Neuropilin-1 extracellular domains mediate semaphorin D/III-induced growth cone collapse. Neuron 21: 1093-1100, 1998

18. Dai DL, Martinka M and Li G: Prognostic significance of activated Akt expression in melanoma: a clinicopathologic study of 292 cases. J Clin Oncol 23: 1473-1482, 2005.

19. Robertson GP: Functional and therapeutic significance of Akt deregulation in malignant melanoma. Cancer Metastasis Rev 24: 273-285, 2005.

20. Kozikowski AP, Sun H, Brognard J and Dennis PA: Novel PI analogues selectively block activation of the pro-survival serine/threonine kinase Akt. J Am Chem Soc 125: 1144-1145, 2003.

21. Wang L, Zeng H, Wang P, Soker S and Mukhopadhyay D: Neuropilin-1-mediated vascular permeability factor/vascular endothelial growth factor-dependent endothelial cell migration. J Biol Chem 278: 48848-48860, 2003. 
22. Murga M, Fernandez-Capetillo O and Tosato G: Neuropilinregulates attachment in human endothelial cells independently of vascular endothelial growth factor receptor-2. Blood 105: 1992-1999, 2005.

23. Cai H and Reed RR: Cloning and characterization of neuropilin1-interacting protein: a PSD-95/Dlg/ZO-1 domain-containing protein that interacts with the cytoplasmic domain of neuropilin-1. J Neurosci 19: 6519-6527, 1999.

24. Chittenden TW, Claes F, Lanahan AA, et al: Selective regulation of arterial branching morphogenesis by synectin. Dev Cell 10: 783-795, 2006.

25. Hofmann UB, Westphal JR, Zendman AJ, Becker JC, Ruiter DJ and van Muijen GNP: Expression and activation of matrix metalloproteinase-2 (MMP-2) and its co-localization with membrane type matrix metalloproteinase 1 (MT1-MMP) correlate with melanoma progression. J Pathol 191: 245-256, 2000.

26. Väisänen A, Kallioinen M, Taskinen PJ and TurpeenniemiHujanen T: Prognostic value of MMP-2 immunoreactive protein (72 kD type IV collagenase) in primary skin melanoma. J Pathol 186: 51-58, 1998

27. Fukasawa M, Matsushita A and Korc M: Neuropilin-1 interacts with integrin betal and modulates pancreatic cancer cell growth, survival and invasion. Cancer Biol Ther 6: 1173-1180, 2007.

28. Westermarck J and Kähäri VM: Regulation of matrix metalloproteinase expression in tumor invasion. FASEB J 13: 781-792, 1999.

29. Park BK, Zeng $X$ and Glazer RI: Akt1 induces extracellular matrix invasion and matrix metalloproteinase- 2 activity in mouse mammary epithelial cells. Cancer Res 61: 7647-7653, 2001.

30. Tremblay F, Brule S, Hee Um S, et al: Identification of IRS-1 Ser-1101 as a target of S6K1 in nutrient and obesity-induced insulin resistance. Proc Natl Acad Sci USA 104: 14056-14061, 2007.

31. Kong M, Fox CJ, Mu J, et al: The PP2A-associated protein alpha 4 is an essential inhibitor of apoptosis. Science 306 : 695-698, 2004.
32. Kuo YC, Huang KY, Yang CH, Yang YS, Lee WY and Chiang CW: Regulation of phosphorylation of Thr-308 of Akt, cell proliferation and survival by the B55alpha regulatory subunit targeting of the protein phosphatase $2 \mathrm{~A}$ holoenzyme to Akt. J Biol Chem 283: 1882-1892, 2008.

33. Steelman LS, Stadelman KM, Chappell WH, et al: Akt as a therapeutic target in cancer. Expert Opin Ther Targets 12: $1139-1165,2008$

34. Guo D, Hildebrandt IJ, Prins RM, et al: The AMPK agonist AICAR inhibits the growth of EGFRvIII-expressing glioblastomas by inhibiting lipogenesis. Proc Natl Acad Sci USA 106: 12932-12937, 2009.

35. Rodrik-Outmezguine V, Chandarlapaty S, Pagano N, et al mTOR kinase inhibition causes feedback-dependent biphasic regulation of AKT signalling. Cancer Discov 3: 248-259, 2011

36. Fenton TR and Gout IT: Functions and regulation of the $70 \mathrm{kDa}$ ribosomal S6 kinases. Int J Biochem Cell Biol 43: 47-59, 2011.

37. Romeo Y, Zhang X and Roux PP: Regulation and function of the RSK family of protein kinases. Biochem J 441: 553-569, 2012.

38. Doehn U, Hauge C, Frank SR, et al: RSK is a principal effector of the RAS-ERK pathway for eliciting a coordinate promotile/ invasive gene program and phenotype in epithelial cells. Mol Cell 35: 511-522, 2009

39. Smolen GA, Zhang J, Zubrowski MJ, et al: A genome-wide RNAi screen identifies multiple RSK-dependent regulators of cell migration. Genes Dev 24: 2654-2665, 2010.

40. Tanimura S, Hashizume J, Kurosaki Y, et al: SH3P2 is a negative regulator of cell motility whose function is inhibited by ribosomal S6 kinase-mediated phosphorylation. Genes Cells 16: 514-526, 2011.

41. Mora A, Komander D, Van Aalten DM and Alessi DR: PDK1, the master regulator of AGC kinase signal transduction. Semin Cell Dev Biol 15: 161-170, 2004 